\title{
Cluster Synchronization for Linearly Coupled Complex Networks with Identical and Nonidentical Nodes
}

\author{
Yi Zhao, Jianwen Feng, and Jingyi Wang \\ College of Mathematics and Computational Science, Shenzhen University, \\ Guangdong Shenzhen 518060, China \\ Correspondence should be addressed to Jianwen Feng, fengjw@szu.edu.cn
}

Received 11 April 2012; Accepted 7 July 2012

Academic Editor: Hai L. Liu

Copyright (C) 2012 Yi Zhao et al. This is an open access article distributed under the Creative Commons Attribution License, which permits unrestricted use, distribution, and reproduction in any medium, provided the original work is properly cited.

\begin{abstract}
The cluster synchronization of linearly coupled complex networks with identical and nonidentical nodes is studied. Without assuming symmetry, we proved that these linearly coupled complex networks could achieve cluster synchronization under certain pinning control schemes. Sufficient conditions guaranteeing cluster synchronization for any initial values are derived by using Lyapunov function methods. Moreover, the adaptive feedback algorithms are proposed to adjust the control strength. Several numerical examples are given to illustrate our theoretical results.
\end{abstract}

\section{Introduction}

Recently, an increasing interest has been devoted to the study of complex networks. Among them, synchronization is the most interesting. In fact, synchronization of complex networks has been found to be a universal phenomenon in nature and has important potential applications in real-world dynamical systems. Great interests and attentions have been received for the synchronization of complex networks in many research and application fields including secure communication, seismology, parallel image processing, chemical reaction, and others [1-7].

There are many widely studied synchronization patterns, such as complete synchronization [8], lag synchronization [9], cluster synchronization [10], phase synchronization [11], and partial synchronization [12] Among them, the studies on cluster synchronization have received more and more attentions. The cluster synchronization requires that the coupled oscillators split into subgroups called clusters, such that the oscillators synchronize with one another in the same cluster, but there is no synchronization among different clusters, 
which could describe the behaviors of the complex network in the real world. For instance, the metabolic, neural, or software networks containing some different function communities. Thus, it is a natural idea to consider the cluster synchronization of such community networks.

The complex network we considered in this paper is the linearly coupled ordinary differential equations (LCODEs). In fact, LCODEs are a large class of dynamical systems with continuous time and state, as well as discrete space, which are widely used to describe coupling oscillators. Nowadays, cluster synchronization of different kinds of LCODEs has been widely studied, and many results have already exist on the various properties of such problem. For instance, Ma et al. [13] constructed a novel coupling scheme with cooperative and competitive weight couplings that guarantees the cluster synchronization of any connected networks with identical nodes. The authors also derived a sufficient condition for the global stability of cluster synchronization. In [14], Wu et al. have discussed the problem of driving linearly coupled networks to an arbitrarily selected cluster synchronization pattern via pinning control. They introduced a single negative feedback controller for each cluster to pin the coupled system to the assigned cluster synchronization pattern for any initial values. However, in most cases, couplings between nodes are not the same even if the diffusive condition is still satisfied. Nodes usually receive instantaneous information as well as delayed information from their neighbors. Thus, it is a nature idea to study the synchronization of the networks with both delayed and nondelayed coupling. In this paper, we would investigate cluster synchronization of LCODEs with both delayed and nondelayed coupling under pinning control scheme. First, we assume all the node in LCODEs are identical. By utilizing the Lyapunov stability method, the global stability of cluster synchronization in networks is investigated, and several sufficient conditions for the global stability are given. Furthermore, we propose an adaptive feedback algorithms to adjust the control strength for LCODEs.

Since in the real world, many networks contain some different function communities and the local dynamics between two function communities are different. For instances, in metabolic, neural, or software community networks, the individual nodes in each community can be viewed as the identical functional units, whereas the nodes in different communities are different since they have different functions [15]. One method to solve such problem is to consider the cluster synchronization of community networks with nonidentical nodes. There have been already some papers focused on sufficient conditions for the global stability of cluster synchronization of some related networks. A number of sufficient conditions were similarly obtained by $\mathrm{Lu}$ et al. [16] for the cluster synchronization of dynamical networks with community structure and nonidentical nodes in the presence of time delays by using a certain feedback control scheme. Lu et al. [17] studied the cluster synchronization of general bi-directed networks with nonidentical clusters and derived sufficient conditions for achieving local cluster synchronization of networks. The authors also discovered a relationship between the cluster synchronizability of a network and its intra-to-intercluster link ratio with the help of numerical examples. Recently, Wang et al. [18] considered the cluster synchronization of dynamical networks with community structure and nonidentical nodes and with identical local dynamics for all individual nodes in each community by using pinning control schemes. In this paper, we also investigate cluster synchronization of LCODEs with nonidentical nodes under pinning control scheme. By utilizing the LyapunovKrasovskii stability method, the global stability of cluster synchronization in networks is investigated, and several sufficient conditions for the global stability are given. Compared with [16-18], the complex network model we considered in this paper is more general. And moreover, the coupling matrices with and without time delay are asymmetric. 
The paper is organized as follows. In Section 2, some necessary and useful definitions and lemmas are given. In Section 3, we study the global cluster synchronization of LCODEs with identical nodes and give a sufficient condition for it. Then, the adaptive feedback algorithms on control strength are proposed to achieve cluster synchronization in the complex network. In Section 4 cluster synchronization of LCODEs with nonidentical nodes is investigated and sufficient conditions are derived to achieve cluster synchronization. And the adaptive feedback algorithms on control strength are also proposed. In Section 5, numerical simulation are presented. We conclude the paper in Section 6.

\section{Preliminaries}

First, we introduce the mathematical definition of cluster synchronization.

Definition 2.1 (see [14]). Let $\left\{U_{1}, \ldots, U_{m}\right\}$ be a partition of the set $\{1,2, \ldots, N\}$ into $d$ nonempty subsets, that is, $U_{l} \neq \phi$ and $\bigcup_{l=1}^{m}=\{1,2, \ldots, N\}$. For $i \in\{1,2, \ldots, N\}$, let $\hat{i}$ denote the subscript of the subset in which the number $i$ is, that is, $i \in U_{\hat{i}}$. A network with $N$ identical oscillators is said to realize $m$-cluster synchronization with the partition $\left\{U_{1}, \ldots, U_{m}\right\}$ if, for any initial values, the state variables of the oscillators satisfy $\lim _{t \rightarrow \infty}\left\|x_{i}(t)-x_{j}(t)\right\|=0$ for $\widehat{i}=\hat{j}$ and $\lim _{t \rightarrow \infty}\left\|x_{i}(t)-x_{j}(t)\right\| \neq 0$ for $\widehat{i} \neq \hat{j}$.

For convenience of the statement to our main results, we now make some definitions for a class of functions and a class of matrices.

Definition 2.2 (see [19-21]). Suppose that $f(x, t)$ is a class of continuous functions $f: R^{n} \times$ $[0, \infty) \rightarrow R^{n}$. Let $P=\operatorname{diag}\left\{p_{1}, p_{2}, \ldots, p_{n}\right\}$ be a positive definite diagonal matrix and $\Delta=$ $\operatorname{diag}\left\{\delta_{1}, \delta_{2}, \ldots, \delta_{n}\right\}$ be a diagonal matrix. $f(x, t) \in \operatorname{QUAD}(P, \Delta, \eta)$ if and only if

$$
(x-y)^{T} P((f(x, t)-f(y, t))-\Delta(x-y)) \leq-\eta(x-y)^{T}(x-y),
$$

holds for some $\eta>0$, where $x, y \in R^{n}$ and $t>0$.

Definition 2.3 (see [14]). For $N \times N$ matrix

$$
A=\left[\begin{array}{cccc}
A_{11} & A_{11} & \ldots & A_{1 d} \\
A_{21} & A_{22} & \ldots & A_{2 d} \\
\vdots & \vdots & \ddots & \vdots \\
A_{d 1} & A_{d 2} & \ldots & A_{d d}
\end{array}\right]
$$

where $A_{u v} \in R^{k_{u} \times k_{v}}, u, v=1,2, \ldots, d$. If each block $A_{u v}$ is a zero-row-sum matrix, then we say that $A \in \mathbf{M}_{\mathbf{1}}(\mathbf{k})$.

For an asymmetric matrix with zero-row-sums, we have the following.

Lemma 2.4 (see [19]). Let $Q$ and $R$ be two symmetric matrices, and matrix $S$ has suitable dimension. Then

$$
\left[\begin{array}{cc}
Q & S \\
S^{T} & R
\end{array}\right]<0
$$

if and only if both $R<0$ and $Q-S R^{-1} S^{T}<0$. 


\section{Cluster Synchronization of LCODEs with Identical Nodes}

The complex network we considered in this section can be described as

$$
\dot{x}_{i}(t)=f\left(x_{i}(t), t\right)+c \sum_{j=1}^{N} a_{i j} x_{j}(t)+c \sum_{j=1}^{N} b_{i j} x_{j}(t-\tau), \quad i=1,2, \ldots, N
$$

where $N$ is the networks size, $x_{i}=\left(x_{1 i}, x_{2 i}, \ldots, x_{n i}\right)^{T} \in R^{n}$ is the state vector of the $i$ th oscillator, $f: R^{n} \times[0, \infty) \rightarrow R^{n}$ is a continues map, and $c>0$ is the coupling strength. $\tau$ is the time delay. $A=\left(a_{i j}\right) \in R^{n \times n}$ and $B=\left(b_{i j}\right) \in R^{n \times n}$ are the coupling configuration matrix with zero-sum rows. It represents the topological structure of the network, in which $a_{i j}>0$ if there is a connection from node $j$ to node $i(i \neq j)$ and is zero otherwise. $B$ has the same properties. Here, $A$ and $B$ need not be symmetric since asymmetric topological structures are most common in the real world.

Without loss of generality, we set the partition of nodes $U_{1}=\left\{1,2, \ldots, k_{1}\right\}, U_{2}=$ $\left\{k_{1}+1, \ldots, k_{1}+k_{2}\right\}, \ldots, U_{m}=\left\{k_{1}+\cdots+k_{m-1}+1, \ldots, k_{1}+\cdots+k_{m-1}+k_{m}\right\}$, where $1<$ $m<N, 1<k_{l}<N$ and $\sum_{l=1}^{m} k_{l}=N$. Let $s_{1}(t), \ldots, s_{m}(t)$ be the $m$ special solutions of the homogenous system $\dot{s}(t)=f(s(t), t)$, which satisfy $\lim _{t \rightarrow \infty}\left\|s_{i}(t)-s_{j}(t)\right\| \neq 0$ for $i \neq j$. By using similar pinning control method in [14], we let the controlled oscillator set $J$ be $J=\left\{k_{1}, k_{1}+k_{2}, \ldots, k_{1}+k_{2}+\cdots+k_{m}\right\}$, which means we only put the control on the last node of each partition $U_{l}$. We use linear negative feedback controllers and the LCODEs (3.1) become

$$
\begin{gathered}
\dot{x}_{i}(t)=f\left(x_{i}(t), t\right)+c \sum_{j=1}^{m} a_{i j} x_{j}(t)+c \sum_{j=1}^{N} b_{i j} x_{j}(t-\tau)-\xi_{i}\left(x_{i}(t)-s_{i}(t)\right), \quad i \in J, \\
\dot{x}_{i}(t)=f\left(x_{i}(t), t\right)+c \sum_{j=1}^{m} a_{i j} x_{j}(t)+c \sum_{j=1}^{N} b_{i j} x_{j}(t-\tau), \quad i \notin J,
\end{gathered}
$$

where $\xi_{u}>0$ with $u \in J$ are the control strengths.

In this section, sufficient conditions are derived for the attainment of cluster synchronization for any initial value by control pinning, that is, by making

$$
\lim _{t \rightarrow \infty}\left\|x_{i}(t)-s_{\hat{i}}(t)\right\|=0 \quad \text { for } \quad i=1,2, \ldots, N
$$

Theorem 3.1. Suppose that the coupling matrices $A$ and $B$ in (3.2) satisfy $A \in \mathbf{M}_{\mathbf{1}}(\mathbf{k})$ and $B \in \mathbf{M}_{\mathbf{1}}(\mathbf{k})$. Let $P=\operatorname{diag}\left\{p_{1}, p_{2}, \ldots, p_{n}\right\}$ be a positive definite diagonal matrix and $\Delta=$ $\operatorname{diag}\left\{\delta_{1}, \delta_{2}, \ldots, \delta_{n}\right\}$ be a diagonal matrix such that $f(x, t) \in \operatorname{QUAD}(P, \Delta, \eta)$. Define $A^{S}=(A+$ $\left.A^{T}\right) / 2$. For $k=1,2, \ldots, n$, if

$$
\left(\Lambda+p_{k} \delta_{k}\right) I+c p_{k} A^{S}-p_{k} \Xi+\frac{c^{2}}{4 \Lambda} p_{k}^{2} B B^{T}<0,
$$


where $\Xi=\operatorname{diag}\left\{\tilde{\xi}_{1}, \tilde{\xi}_{2}, \ldots, \tilde{\xi}_{N}\right\}$ satisfies

$$
\widetilde{\xi}_{i}= \begin{cases}\xi_{i}, & i \in J \\ 0, & i \notin J .\end{cases}
$$

Then, for any initial values, the solution $x_{1}(t), x_{2}(t), \ldots, x_{N}(t)$ of the system (3.1) under the control (3.2) can achieve cluster synchronization and satisfies (3.3).

Proof. We define $e_{i}(t)=x_{i}(t)-s_{\hat{i}}(t)$, where $i=1,2, \ldots, N$. Denote $e^{k}(t)=$ $\left(e_{k 1}(t), e_{k 2}(t), \ldots, e_{k N}(t)\right)^{T}$ for $k=1,2, \ldots n$. Since $A \in \mathbf{M}_{1}(\mathbf{k})$, which means that $\sum_{i \in U_{l}} a_{i j}=0$ hold for all $i=1,2, \ldots, N$ and $l=1,2, \ldots, m$. It is readily seen that

$$
\begin{aligned}
\sum_{j=1}^{N} a_{i j} x_{j} & =\sum_{l=1}^{m} \sum_{j \in U_{l}} a_{i j} x_{j}=\sum_{l=1}^{m} \sum_{j \in U_{l}} a_{i j}\left(x_{j}-s_{l}+s_{l}\right) \\
& =\sum_{l=1}^{m} \sum_{j \in U_{l}} a_{i j} e_{j}+\sum_{l=1}^{m} \sum_{j \in U_{l}} a_{i j} s_{l}=\sum_{j=1}^{N} a_{i j} e_{j} .
\end{aligned}
$$

And similarly, $\sum_{j=1}^{N} b_{i j} x_{j}=\sum_{j=1}^{N} b_{i j} e_{j}$. Thus, $e_{i}(t)$ for $i=1, \ldots N$ satisfies the following differential equation:

$$
\begin{gathered}
\dot{e}_{i}(t)=f\left(x_{i}(t), t\right)-f\left(s_{\hat{i}}(t), t\right)+c \sum_{j=1}^{m} a_{i j} e_{j}(t)+c \sum_{j=1}^{N} b_{i j} e_{j}(t-\tau)-\xi_{i} e_{i}(t), \quad i \in J, \\
\dot{e}_{i}(t)=f\left(x_{i}(t), t\right)-f\left(s_{\hat{i}}(t), t\right)+c \sum_{j=1}^{m} a_{i j} e_{j}(t)+c \sum_{j=1}^{N} b_{i j} e_{j}(t-\tau), \quad i \notin J,
\end{gathered}
$$

Choose a Lyapunov function as

$$
V(t)=\frac{1}{2} \sum_{i=1}^{N}\left(e_{i}(t)^{T} P e_{i}(t)+2 \Lambda \int_{t-\tau}^{t} e_{i}(\theta)^{T} e_{i}(\theta) d \theta\right)
$$


Note that $f(x, t) \in \operatorname{QUAD}(P, \Delta, \eta)$. We differentiate (3.8) along (3.7) and have

$$
\begin{aligned}
& \frac{d V}{d t}= \sum_{i=1}^{N} e_{i}(t)^{T} P \dot{e}_{i}(t)+\Lambda \sum_{i=1}^{N} e_{i}(t)^{T} e_{i}(t)-\Lambda \sum_{i=1}^{N} e_{i}^{T}(t-\tau) e_{i}(t-\tau) \\
&= \sum_{i=1}^{N} e_{i}(t)^{T} P\left[f\left(x_{i}(t), t\right)-f\left(s_{i}(t), t\right)+c \sum_{j=1}^{N} a_{i j} e_{j}(t)+c \sum_{j=1}^{N} b_{i j} e_{j}(t-\tau)\right] \\
&-\sum_{i \in J} e_{i}(t)^{T} P \xi_{i} e_{i}(t)+\Lambda \sum_{i=1}^{N} e_{i}(t)^{T} e_{i}(t)-\Lambda \sum_{i=1}^{N} e_{i}^{T}(t-\tau) e_{i}(t-\tau) \\
& \leq-\eta \sum_{i=1}^{N} e_{i}(t)^{T} e_{i}(t)+\sum_{i=1}^{N} e_{i}(t)^{T} P \Delta e_{i}(t)+c \sum_{i=1}^{N} e_{i}(t)^{T} P \sum_{j=1}^{N} a_{i j} e_{j}(t) \\
&+c \sum_{i=1}^{N} e_{i}(t)^{T} P \sum_{j=1}^{N} b_{i j} e_{j}(t-\tau)-\sum_{i \in J} \xi_{i} e_{i}(t)^{T} P e_{i}(t) \\
&+\Lambda \sum_{i=1}^{N} e_{i}(t)^{T} e_{i}(t)-\Lambda \sum_{i=1}^{N} e_{i}^{T}(t-\tau) e_{i}(t-\tau) \\
&=-\eta \sum_{i=1}^{N} e_{i}(t)^{T} e_{i}(t)+\sum_{k=1}^{n} p_{k} \delta_{k} e^{k}(t)^{T} e^{k}(t)+c \sum_{k=1}^{n} p_{k} e^{k}(t)^{T} A^{S} e^{k}(t) \\
&-\sum_{k=1}^{n} p_{k} e^{k}(t)^{T} \Xi e^{k}(t)+c \sum_{k=1}^{n} p_{k} e^{k}(t)^{T} B e^{k}(t-\tau) \\
&+\Lambda \sum_{k=1}^{n} e^{k}(t)^{T} e^{k}(t)-\Lambda \sum_{i=1}^{n} e^{k}(t-\tau)^{T} e^{k}(t-\tau) \\
&=-\eta \sum_{i=1}^{N} e_{i}(t)^{T} e_{i}(t)+\sum_{k=1}^{n}\left(e^{k}(t)^{T}, e^{k}(t-\tau)^{T}\right) \\
& \times\left[\left(\Lambda+p_{k} \delta_{k}\right) I+c p_{k} A^{S}-p_{k} \Xi \frac{c}{2} p_{k} B\right]\left(e^{k}(t), e^{k}(t-\tau)\right) \\
&- {[-\Lambda I] } \\
& p_{k} B^{T}
\end{aligned}
$$

where $\Xi$ is defined in (3.5).

If (3.4) is satisfied, from Lemma 2.4, it can be easily seen that $d V / d t<0$, and thus (3.2) could achieve cluster synchronization.

By using adaptive adjustments, we can find relatively small control strength to realize cluster synchronization. We regard the control strength of the network functions varying with time. Then, we could design the adaptive control strength. Then, we have the following result. 
Theorem 3.2. Suppose that the coupling matrix $A$ and $B$ in (3.2) satisfy $A \in \mathbf{M}_{\mathbf{1}}(\mathbf{k})$ and $B \in \mathbf{M}_{\mathbf{1}}(\mathbf{k})$. The control strength $\xi_{i}$ in (3.2) is defined as

$$
\dot{\xi}_{i}(t)=\gamma_{i} e_{i}^{T}(t) P e_{i}(t) \quad \text { for } i \in J,
$$

where $\gamma_{i}$ is a positive constant. Let $P=\operatorname{diag}\left\{p_{1}, p_{2}, \ldots, p_{n}\right\}$ be a positive definite diagonal matrix and $\Delta=\operatorname{diag}\left\{\delta_{1}, \delta_{2}, \ldots, \delta_{n}\right\}$ be a diagonal matrix such that $f(x, t) \in \operatorname{QUAD}(P, \Delta, \eta)$. For $k=$ $1,2, \ldots, n$, if

$$
\left(\Lambda+p_{k} \delta_{k}\right) I+c p_{k} A^{S}-p_{k} \Xi^{*}+\frac{c^{2}}{4 \Lambda} p_{k}^{2} B B^{T}<0
$$

where $\Xi^{*}=\operatorname{diag}\left\{\xi_{1}^{*}, \xi_{2}^{*}, \ldots, \xi_{N}^{*}\right\} . \xi_{i}^{*}$ is a constant and satisfies $\xi_{i}^{*}=0$ if $i \notin J$. Then, for any initial values, the solution $x_{1}(t), x_{2}(t), \ldots, x_{N}(t)$ of the system (3.1) under the control (3.2) can achieve cluster synchronization and satisfies (3.3).

Proof. Choose a Lyapunov function as

$$
V(t)=\frac{1}{2} \sum_{i=1}^{N}\left(e_{i}(t)^{T} P e_{i}(t)+2 \Lambda \int_{t-\tau}^{t} e_{i}(\theta)^{T} e_{i}(\theta) d \theta\right)+\sum_{i \in J} \frac{\left(\xi_{i}-\xi_{i}^{*}\right)^{2}}{2 \gamma_{i}}
$$

Note that $f(x, t) \in \operatorname{QUAD}(P, \Delta, \eta)$. We differentiate (3.12) along (3.7) and have

$$
\begin{aligned}
\frac{d V}{d t}= & \sum_{i=1}^{N} e_{i}(t)^{T} P \dot{e}_{i}(t)+\Lambda \sum_{i=1}^{N} e_{i}(t)^{T} e_{i}(t)-\Lambda \sum_{i=1}^{N} e_{i}^{T}(t-\tau) e_{i}(t-\tau) \\
& +\sum_{i \in J}\left(\xi_{i}-\xi_{i}^{*}\right) e_{i}^{T}(t) P e_{i}(t) \\
= & \sum_{i=1}^{N} e_{i}(t)^{T} P\left[f\left(x_{i}(t), t\right)-f\left(s_{\hat{i}}(t), t\right)+c \sum_{j=1}^{N} a_{i j} e_{j}(t)+c \sum_{j=1}^{N} b_{i j} e_{j}(t-\tau)\right] \\
& -\sum_{i \in J} e_{i}(t)^{T} P \xi_{i}^{*} e_{i}(t)+\Lambda \sum_{i=1}^{N} e_{i}(t)^{T} e_{i}(t)-\Lambda \sum_{i=1}^{N} e_{i}^{T}(t-\tau) e_{i}(t-\tau) .
\end{aligned}
$$

By using similar calculations in (3.9), we have

$$
\begin{aligned}
\frac{d V}{d t} \leq & -\eta \sum_{i=1}^{N} e_{i}(t)^{T} e_{i}(t)+\sum_{k=1}^{n}\left(e^{k}(t)^{T}, e^{k}(t-\tau)^{T}\right) \\
& \times\left[\begin{array}{cc}
\left(\Lambda+p_{k} \delta_{k}\right) I+c p_{k} A^{S}-p_{k} \Xi^{*} & \frac{c}{2} p_{k} B \\
\frac{c}{2} p_{k} B^{T} & -\Lambda I
\end{array}\right]\left(e^{k}(t), e^{k}(t-\tau)\right) .
\end{aligned}
$$

Noticing the inequalities in (3.11), we could obtain $d V(t) / d t<0$. Thus, we get $x_{i}(t) \rightarrow s_{\hat{i}}(t)$, and $\dot{\xi}_{i}(t) \rightarrow 0$. By Cauchy convergence principle, $\xi_{i}(t)$ converges. 


\section{Cluster Synchronization of LCODEs with Nonidentical Nodes}

The complex network considered in this section is

$$
\dot{x}_{i}(t)=f_{\mu_{i}}\left(x_{i}(t), t\right)+c \sum_{j=1}^{N} a_{i j} x_{j}(t)+c \sum_{j=1}^{N} b_{i j} x_{j}(t-\tau), \quad i=1,2, \ldots, N,
$$

where $x_{i}=\left(x_{1 i}, x_{2 i}, \ldots, x_{n i}\right)^{T} \in R^{n}$ are the state variables of node $i$. The complex network (4.1) has $N$ nodes and $m$ communities with $N>m \geq 2$. If node $i$ belongs to the $j$ th community, then we let $\mu_{i}=j$. We denote by $U_{i}$ the set of all nodes in the $i$ th community and let $\tilde{U}_{i}^{A}$, which is the subset of $U_{i}$, be the index set of all nodes in the $i$ th community having direct connections to other communities in $A$. And by the similar way, we can define $\tilde{U}_{i}^{B}$. The function $f_{\mu_{i}}(\cdot)$ describes the local dynamics of nodes in the $\mu_{i}$ th community, which is differentiable and capable of performing abundant dynamical behaviors. For any pair of indices $i$ and $j$, if $\mu_{i} \neq \mu_{j}$, which means that node $i$ and node $j$ belong to different communities, then $f_{\mu_{i}} \neq f_{\mu_{j}}$. The constant $c>0$ denotes the coupling strength. $\tau$ is the time delay in couplings. For $A=\left(a_{i j}\right) \in R^{n \times n}$ and $B=\left(b_{i j}\right) \in R^{n \times n}$ are the coupling configuration matrices with zerosum rows, which represent the topological structure of the network. Take $A$ for an example and $B$ has the same properties. $a_{i j}>0$ if there is a connection and is zero otherwise. Also, $A$ and $B$ need not be symmetric.

Let $u_{i}(t) \in R^{n}, i=1, \ldots, N$, be the control inputs, then the controlled dynamical network with respect to (4.1) can be described by

$$
\dot{x}_{i}(t)=f_{\mu_{i}}\left(x_{i}(t), t\right)+c \sum_{j=1}^{N} a_{i j} x_{j}(t)+c \sum_{j=1}^{N} b_{i j} x_{j}(t-\tau)+u_{i}(t), \quad i=1,2, \ldots, N .
$$

We define the error variables by $e_{i}(t)=x_{i}(t)-s_{\mu_{i}}(t)$ for $i=1,2, \ldots, N$, where $s_{\mu_{i}}=$ $\left(s_{1 \mu_{i}}, s_{2 \mu_{i}}, \ldots, s_{n \mu_{i}}\right)^{T} \in R^{n}$ satisfies $\dot{s}_{\mu_{i}}(t)=f_{\mu_{i}}\left(s_{\mu_{i}}(t)\right)$, which describes the identical local dynamics for the nodes in the $\mu_{i}$ th community. The $N$ nodes are said to achieve cluster synchronization if

$$
\lim _{t \rightarrow \infty}\left\|e_{i}(t)\right\|=0, \quad i=1, \ldots, N,
$$

which means that the nodes within $\mu_{i}$ th community are fully synchronized to dynamic state $s_{\mu_{i}}$, while nodes in separate communities behave independently. Define a set $M=$ $\left(s_{\mu_{1}}, s_{\mu_{2}}, \ldots, s_{\mu_{N}}\right) \in R^{n \times N}$ as the cluster synchronization manifold for network (4.2). In fact, condition (4.3) implies that the manifold $M$ is stable.

According to the above definition of error variables, we can write the corresponding error system with respect to (4.2) as

$$
\begin{aligned}
\dot{e}_{i}(t)= & f_{\mu_{i}}\left(x_{i}(t)\right)-f_{\mu_{i}}\left(s_{\mu_{i}}(t)\right)+c \sum_{j=1}^{N} a_{i j} e_{j}(t)+c \sum_{j=1}^{N} a_{i j} s_{\mu_{j}}(t) \\
& +c \sum_{j=1}^{N} b_{i j} e_{j}(t-\tau)+c \sum_{j=1}^{N} b_{i j} s_{\mu_{j}}(t-\tau)+u_{i}(t), \quad i=1, \ldots, N .
\end{aligned}
$$


Since $A$ and $B$ are zero-row-sum matrices, we have

$$
\begin{aligned}
& \sum_{j=1}^{N} a_{i j} s_{\mu_{j}}(t)=0, \quad i \in U_{i} \backslash \tilde{U}_{\mu_{i}}^{A}, \\
& \sum_{j=1}^{N} b_{i j} s_{\mu_{j}}(t)=0, \quad i \in U_{i} \backslash \tilde{U}_{\mu_{i}}^{B} .
\end{aligned}
$$

Let $d_{i}$ stands for the feedback control strength. We design a local feedback control as

$$
u_{i}(t)= \begin{cases}l-c d_{i}^{A} e_{i}(t)-c \sum_{j=1}^{N} a_{i j} s_{\mu_{j}}(t)-c d_{i}^{B} e_{i}(t-\tau)-c \sum_{j=1}^{N} b_{i j} s_{\mu_{j}}(t-\tau), & i \in \tilde{U}_{\mu_{i}}^{A} \cup \tilde{U}_{\mu_{i}}^{B} \\ 0, & \text { otherwise }\end{cases}
$$

where $d_{i}^{A}=d_{i}>0$ for $i \in \tilde{U}_{\mu_{i}}^{A}$ and $d_{i}^{B}=d_{i}>0$ for $i \in \tilde{U}_{\mu_{i}}^{B}$, which means we put control on the nodes that have communications with nodes in other different clusters. By intuition, the terms $c d_{i}^{A} e_{i}(t)$ and $c d_{i}^{B} e_{i}(t-\tau)$ in (4.6) is used to synchronize all nodes in the same cluster, while the remainder terms $c \sum_{j=1}^{N} a_{i j} s_{\mu_{j}}(t)$ and $c \sum_{j=1}^{N} b_{i j} s_{\mu_{j}}(t-\tau)$ in the controller is to weaken the mutual influences among clusters at the intersection nodes. It is easy to verify that the manifold $M$ is an invariant manifold for the network (4.2).

Theorem 4.1. Let $P=\operatorname{diag}\left\{p_{1}, p_{2}, \ldots, p_{n}\right\}$ be a positive definite diagonal matrix and $\Delta=$ $\operatorname{diag}\left\{\delta_{1}, \delta_{2}, \ldots, \delta_{n}\right\}$ be a diagonal matrix such that $f_{\mu_{i}}(x, t) \in \operatorname{QUAD}(P, \Delta, \eta)$ for $1 \leq i \leq N$. For $k=1,2, \ldots, n$, if

$$
\left(\Lambda+p_{k} \delta_{k}\right) I+c p_{k}\left(A^{S}-D_{A}\right)+\frac{c^{2}}{4 \Lambda} p_{k}^{2}\left(B-D_{B}\right)\left(B-D_{B}\right)^{T}<0
$$

where $D_{A}=\operatorname{diag}\left\{d_{1}^{A}, d_{2}^{A}, \ldots, d_{N}^{A}\right\}$ and $D_{B}=\operatorname{diag}\left\{d_{1}^{B}, d_{2}^{B}, \ldots, d_{N}^{B}\right\} . \Lambda$ is a given positive constant Then, for any initial values, the solution $x_{1}(t), x_{1}(t), \ldots, x_{N}(t)$ of the system (4.1) under the control (4.6) can achieve cluster synchronization and satisfies (4.3).

Proof. Denote $e^{k}(t)=\left(e_{k 1}(t), e_{k 2}(t), \ldots, e_{k N}(t)\right)^{T}, k=1,2, \ldots, n$. Define a LyapunovKrasovskii function as

$$
V(t)=\frac{1}{2} \sum_{i=1}^{N}\left\{e_{i}(t)^{T} P e_{i}(t)+2 \Lambda \int_{t-\tau}^{t} e_{i}(\theta)^{T} e_{i}(\theta) d \theta\right\} .
$$


Differentiating (4.8) along the solution of error system (4.4) under the control (4.6) gives

$$
\begin{aligned}
& \frac{d V(t)}{d t}=\sum_{i=1}^{N} e_{i}(t)^{T} P \dot{e}_{i}(t)+\Lambda \sum_{i=1}^{N} e_{i}(t)^{T} e_{i}(t)-\Lambda \sum_{i=1}^{N} e_{i}(t-\tau)^{T} e_{i}(t-\tau) \\
& =\sum_{i=1}^{N} e_{i}(t)^{T} P\left(f_{\mu_{i}}\left(x_{i}(t)\right)-f_{\mu_{i}}\left(s_{\mu_{i}}(t)\right)+c \sum_{j=1}^{N} a_{i j} e_{j}(t)\right) \\
& -\sum_{i=1}^{N} c d_{i}^{A} e_{i}(t)^{T} P e_{i}(t)+\sum_{i=1}^{N} e_{i}(t)^{T} P c \sum_{j=1}^{N} b_{i j} e_{j}(t-\tau)-\sum_{i=1}^{N} c d_{i}^{B} e_{i}(t)^{T} P e_{j}(t-\tau) \\
& +\Lambda \sum_{i=1}^{N} e_{i}(t)^{T} e_{i}(t)-\Lambda \sum_{i=1}^{N} e_{i}(t-\tau)^{T} e_{i}(t-\tau) \\
& =\sum_{i=1}^{N} e_{i}(t)^{T} P\left(f_{\mu_{i}}\left(x_{i}(t)\right)-f_{\mu_{i}}\left(s_{\mu_{i}}(t)\right)-\Delta e_{i}(t)\right)+\sum_{i=1}^{N} e_{i}(t)^{T} P \Delta e_{i}(t) \\
& +\sum_{i=1}^{N} e_{i}(t)^{T} P c \sum_{j=1}^{N} a_{i j} e_{j}(t)-\sum_{i=1}^{N} c d_{i}^{A} e_{i}(t)^{T} P e_{i}(t)+\sum_{i=1}^{N} e_{i}(t)^{T} P c \sum_{j=1}^{N} b_{i j} e_{j}(t-\tau) \\
& -\sum_{i=1}^{N} c d_{i}^{B} e_{i}(t)^{T} P e_{j}(t-\tau)+\Lambda \sum_{i=1}^{N} e_{i}(t)^{T} e_{i}(t)-\Lambda \sum_{i=1}^{N} e_{i}(t-\tau)^{T} e_{i}(t-\tau) \\
& \leq-\eta \sum_{i=1}^{N} e_{i}(t)^{T} e_{i}(t)+\sum_{k=1}^{n} p_{k} \delta_{k} e^{k}(t)^{T} e^{k}(t)+c \sum_{k=1}^{n} p_{k} e^{k}(t)^{T} A^{S} e^{k}(t) \\
& -c \sum_{k=1}^{n} p_{k} e^{k}(t)^{T} D_{A} e^{k}(t)+c \sum_{k=1}^{n} p_{k} e^{k}(t)^{T}\left(B-D_{B}\right) e^{k}(t-\tau) \\
& +\Lambda \sum_{i=1}^{N} e_{i}(t)^{T} e_{i}(t)-\Lambda \sum_{i=1}^{N} e_{i}(t-\tau)^{T} e_{i}(t-\tau) \\
& =-\eta \sum_{i=1}^{N} e_{i}(t)^{T} e_{i}(t)+\sum_{k=1}^{n} p_{k} e^{k}(t)^{T}\left(\delta_{k} I_{N}+c\left(A^{S}-D_{A}\right)\right) e^{k}(t) \\
& +c \sum_{k=1}^{n} p_{k} e^{k}(t)^{T}\left(B-D_{B}\right) e^{k}(t-\tau)+\Lambda \sum_{i=1}^{N} e_{i}(t)^{T} e_{i}(t) \\
& -\Lambda \sum_{i=1}^{N} e_{i}(t-\tau)^{T} e_{i}(t-\tau) \\
& =-\eta \sum_{i=1}^{N} e_{i}(t)^{T} e_{i}(t)+\sum_{k=1}^{n}\left(e^{k}(t)^{T}, e^{k}(t-\tau)^{T}\right) \\
& \times\left[\begin{array}{cc}
\left(\Lambda+p_{k} \delta_{k}\right) I+c p_{k}\left(A^{S}-D_{A}\right) & \frac{c}{2} p_{k}\left(B-D_{B}\right) \\
\frac{c}{2} p_{k}\left(B-D_{B}\right)^{T} & -\Lambda I
\end{array}\right]\left(e^{k}(t), e^{k}(t-\tau)\right) .
\end{aligned}
$$




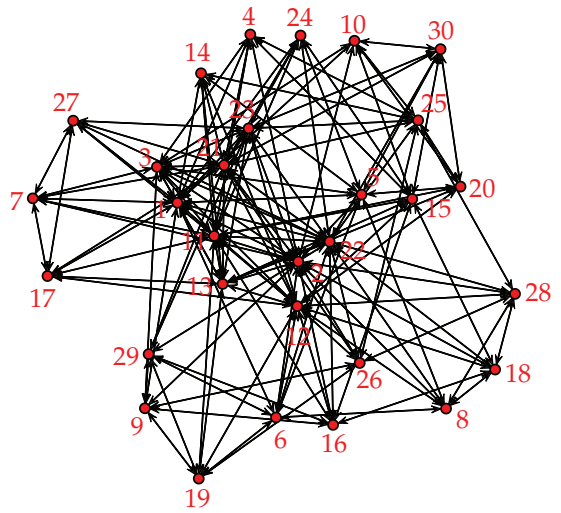

(a)

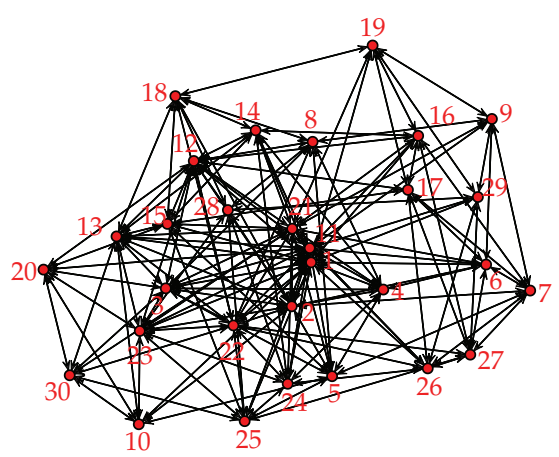

(c)

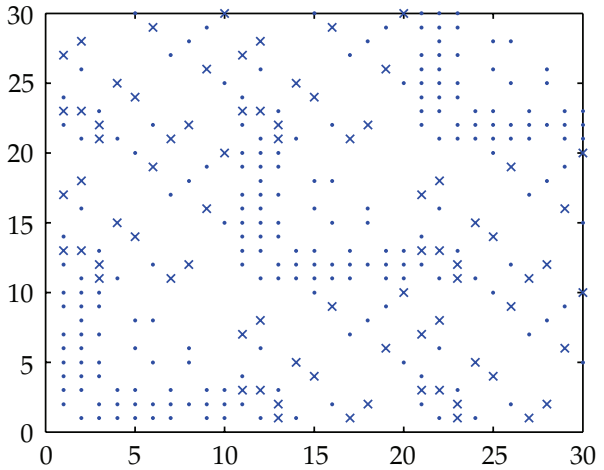

(b)

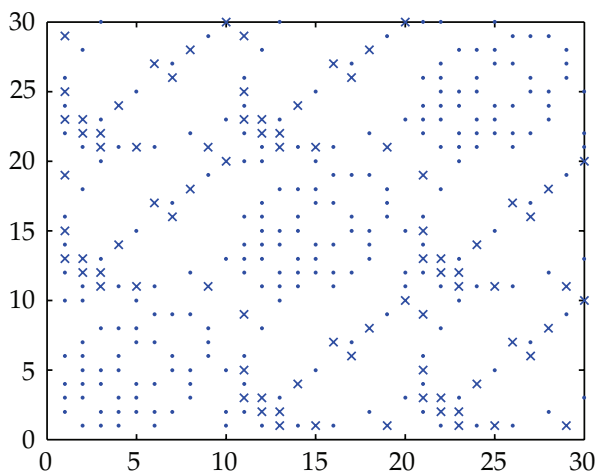

(d)

Figure 1: The controlled complex network (3.2). (a) the topological structure of matrix $A$; (b) The sketch of matrix $A$ : the point $(i, j)$ is denoted as "." if $a_{i j}>0$ and the point $(i, j)$ is denoted as " ${ }^{\prime \prime}$ if $a_{i j}<0$; (c) the topological structure of matrix $B ;(\mathrm{d})$ the sketch of matrix $B$ : the point $(i, j)$ is denoted as "." if $b_{i j}>0$ and the point $(i, j)$ is denoted as " $\times$ " if $b_{i j}<0$.

Noticing the inequalities of (4.7), from Lemma 2.4, we obtain

$$
\frac{d V(t)}{d t} \leq-\eta \sum_{i=1}^{N} e_{i}(t)^{T} e_{i}(t)<0 .
$$

Thus, we get $x_{i}(t) \rightarrow s_{\mu_{i}}(t)$.

\section{Numerical Simulation}

In this section, we give numerical simulations to verify the theorems obtained in Section 3 and Section 4. Consider the unified system,

$$
f(x, t, \alpha)=\left\{\begin{array}{l}
(25 \alpha+10)\left(x_{2}-x_{1}\right) \\
(28-35 \alpha) x_{1}-x_{1} x_{3}+(29 \alpha-1) x_{2} \\
x_{1} x_{2}-\frac{8+\alpha}{3} x_{3}
\end{array}\right.
$$




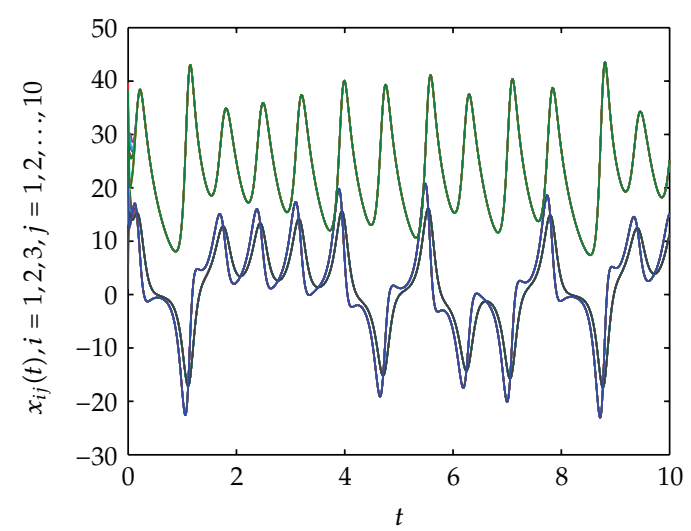

(a)

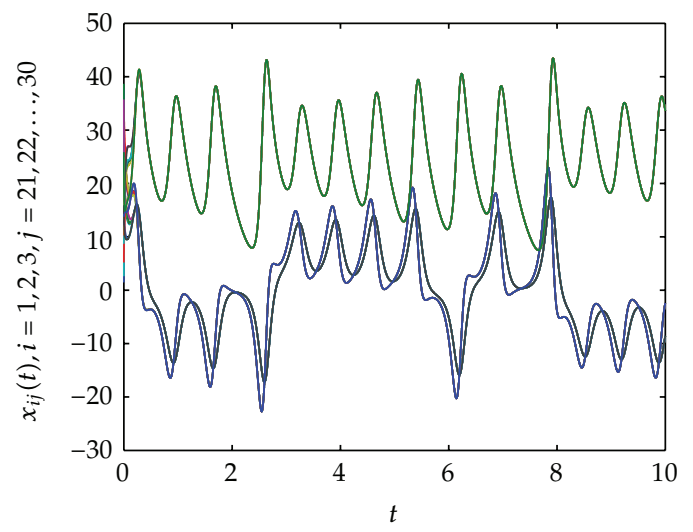

(c)

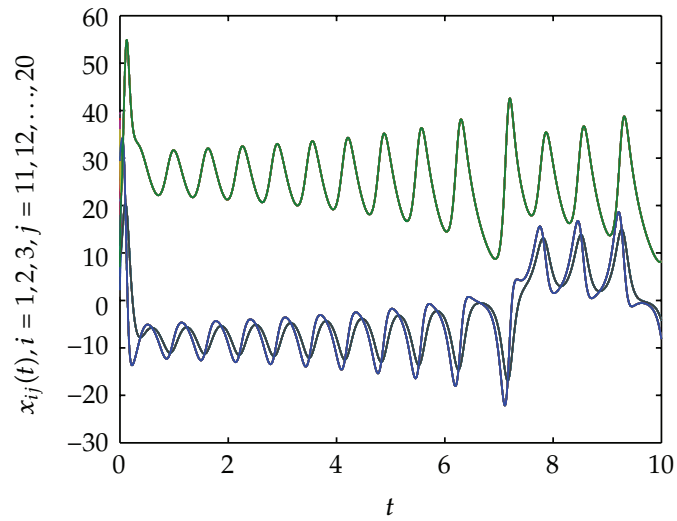

(b)

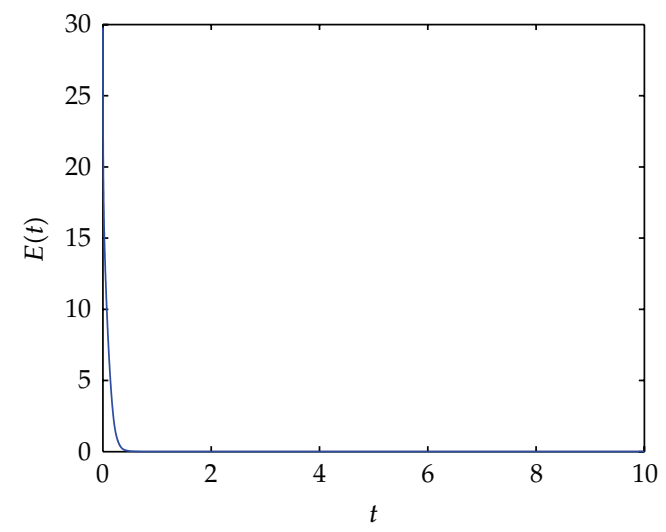

(d)

Figure 2: The controlled complex network (3.2). (a, b, c) The behavior of $x(t)$ in 3 communities. (d) The error $E(t)$.

The system is chaotic for all $\alpha \in[0,1]$. If $\alpha=0$, the system (5.1) is Lorenz's attractor. The ultimate bound and positively invariant set for system (5.1) is given in [22]. For $0 \leq \alpha<1 / 29$, $x_{2}^{2}+\left(x_{3}-28+35 \alpha\right)^{2} \leq C^{2}$ and $x_{1}^{2} \leq C^{2}$, where

$$
C=\frac{(28-35 \alpha)(8+\alpha)}{2 \sqrt{3(5+88 \alpha)(1-29 \alpha)}}
$$

Thus, we have $\left|x_{1}\right| \leq C,\left|x_{2}\right| \leq C$ and $\left|x_{3}\right| \leq C+28-35 \alpha$. Let $P=\operatorname{diag}\left\{p_{1}, p_{2}, p_{3}\right\}$ and $x-s=$ $\left(e_{1}, e_{2}, e_{3}\right)^{T}$, we obtain

$$
\begin{aligned}
(x- & s)^{T} P(f(x, t, \alpha)-f(s, t, \alpha)) \\
\leq & -(25 \alpha+10) p_{1} e_{1}^{2}+(29 \alpha-1) p_{2} e_{2}^{2}-\frac{8+\alpha}{3} p_{3} e_{3}^{2} \\
& +\left((25 \alpha+10) p_{1}+(28-35 \alpha) p_{2}+p_{2}(C+28-35 \alpha)\right)\left|e_{1} e_{2}\right| \\
& +p_{3} C\left|e_{1} e_{3}\right|+\left(p_{3}-p_{2}\right) x_{1} e_{2} e_{3}+\left(p_{2}-p_{3}\right) e_{1} e_{2} e_{3} .
\end{aligned}
$$




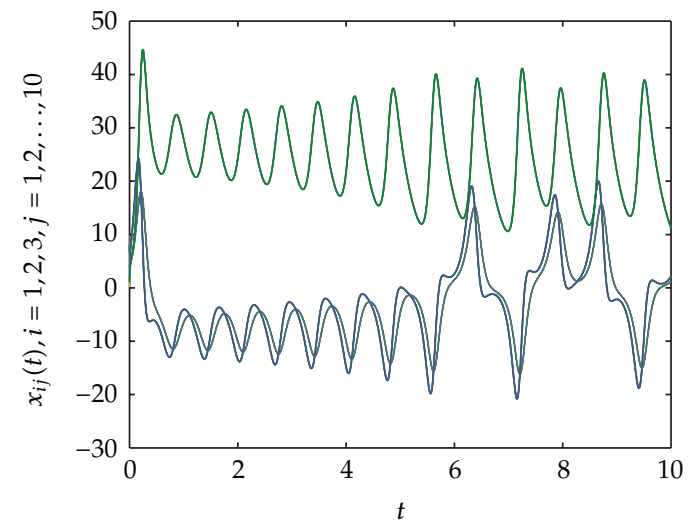

(a)

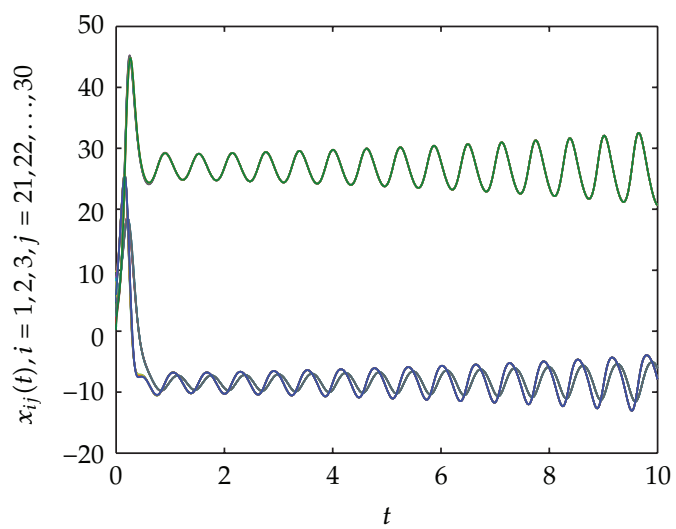

(c)

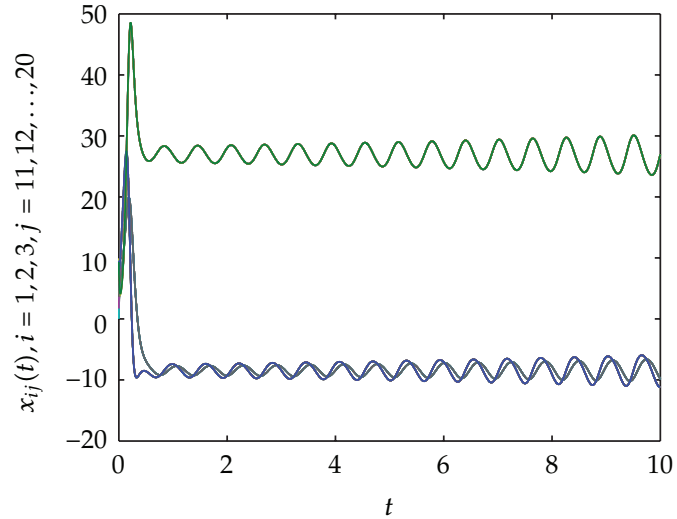

(b)

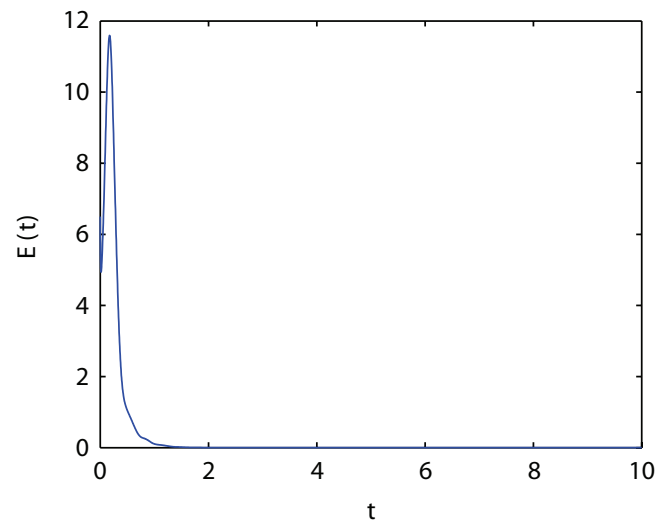

(d)

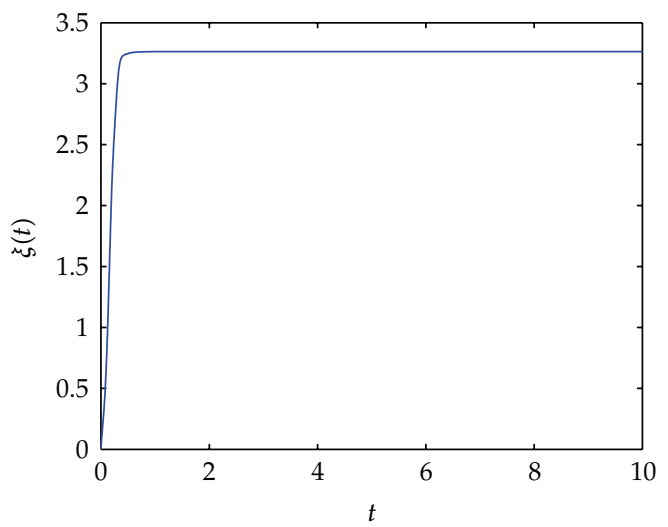

(e)

Figure 3: The controlled complex network (3.2). (a,b,c) The behavior of $x(t)$ in 3 communities. (d) The error $E(t)$. (e) The adaptive control strength $\xi(\mathrm{t})$. 


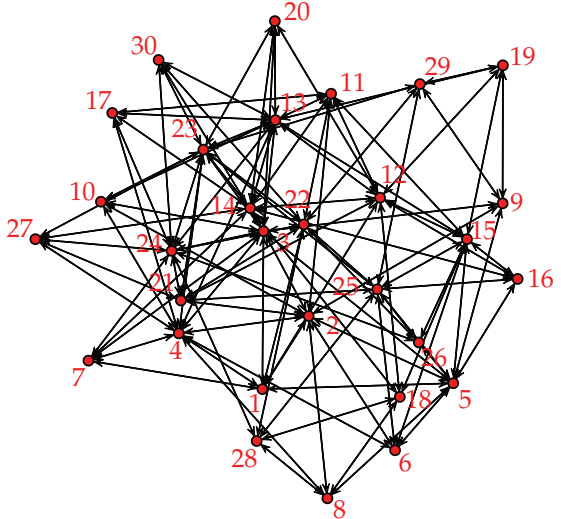

(a)

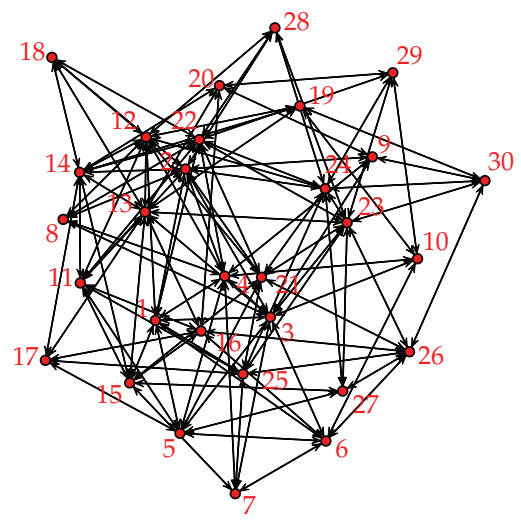

(c)

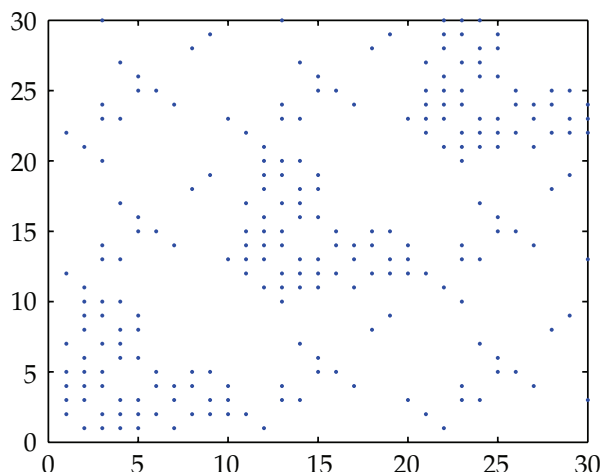

(b)

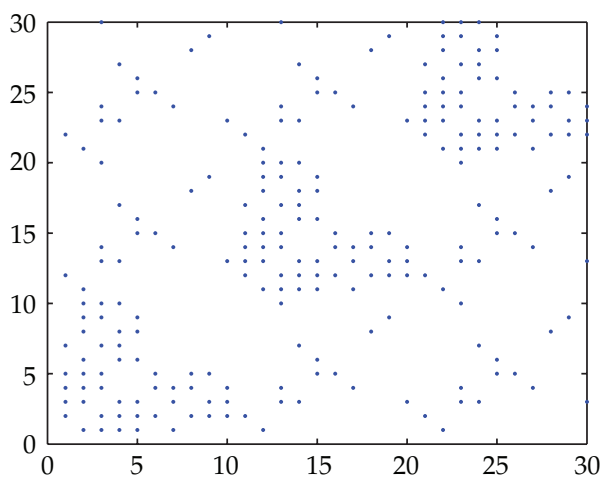

(d)

Figure 4: The controlled complex network (3.2). (a) The topological structure of matrix $A$; (b) the sketch of matrix $A$ : the point $(i, j)$ is denoted as "." if $a_{i j}>0$; (c) the topological structure of matrix $B$; (d) the sketch of matrix $B$ : the point $(i, j)$ is denoted as "." if $b_{i j}>0$.

Let $p_{2}=p_{3}$, we have

$$
\begin{array}{rl}
(x-s)^{T} & P(f(x, t, \alpha)-f(s, t, \alpha)) \\
\leq & \left(-(25 \alpha+10) p_{1}+\frac{p_{3} C}{2} \vartheta+\frac{(25 \alpha+10) p_{1}+(28-35 \alpha) p_{2}+p_{2}(C+28-35 \alpha)}{2} v\right) e_{1}^{2} \\
& +\left((29 \alpha-1) p_{2}+\frac{(25 \alpha+10) p_{1}+(28-35 \alpha) p_{2}+p_{2}(C+28-35 \alpha)}{2 v}\right) e_{2}^{2} \\
& +\left(-\frac{8+\alpha}{3} p_{3}+\frac{p_{3} C}{2 \vartheta}\right) e_{3}^{2} \\
\leq & \left(p_{1} \delta_{1}-\eta\right) e_{1}^{2}+\left(p_{2} \delta_{2}-\eta\right) e_{2}^{2}+\left(p_{3} \delta_{3}-\eta\right) e_{3}^{2} .
\end{array}
$$

Set $P=\operatorname{diag}\{5,1,1\}, \Delta=\operatorname{diag}\{23,36,17\}, \varepsilon=2$ and $\vartheta=1$. Let $\alpha=0, \alpha=0.01$ and $\alpha=0.03$ respectively in (5.1) since the nodes are nonidentical in different communities. 


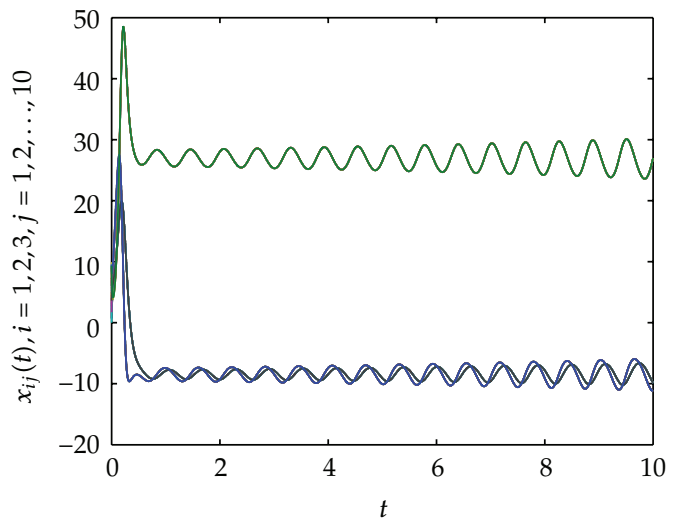

(a)

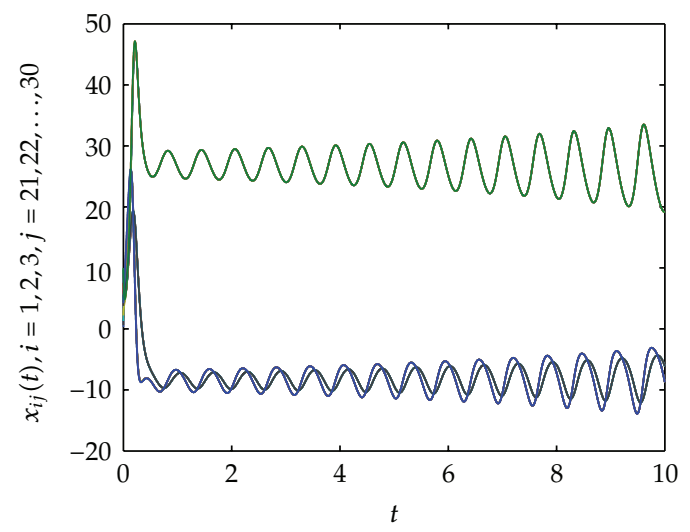

(c)

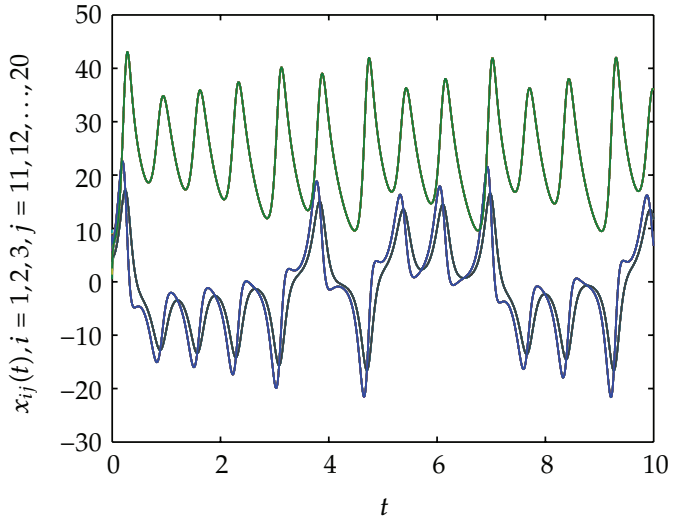

(b)

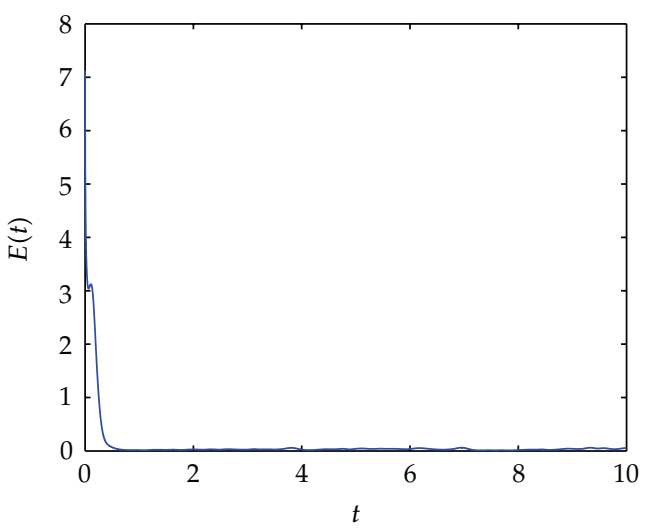

(d)

Figure 5: The controlled complex network (3.2). (a, b, c) The behavior of $x(t)$ in 3 communities. (d) The error $E(t)$.

If $\alpha=0$, we have $\eta_{1}=3.2074$ and $f(x, 0) \in \operatorname{QUAD}\left(P, \Delta, \eta_{1}\right)$. If $\alpha=0.01$, we have $\eta_{2}=2.0243$ and $f(x, 0.01) \in \operatorname{QUAD}\left(P, \Delta, \eta_{2}\right)$. If $\alpha=0.03$, we have $\eta_{3}=0.1547$ and $f(x, 0.02) \in \operatorname{QUAD}\left(P, \Delta, \eta_{3}\right)$.

\subsection{Simulation to Cluster Synchronization with Identical Nodes}

In this simulation, we consider a network with 30 nodes and 3 communities. It is too high and we do not show it out. We show the topology structure in Figure 1.

Let $s_{1}(t), s_{2}(t), s_{3}(t)$ be the solution of the uncoupling system $\dot{s}_{i}(t)=f\left(s_{i}(t), t\right)$ with initial values $s_{1}(0)=[1,2,3]^{T}, s_{2}(0)=[4,5,6]^{T}$, and $s_{3}(0)=[7,8,9]^{T}$. Define $E(t)=(1 / 30) \sum_{i=1}^{30}\left\|x_{i}(t)-s_{i}(t)\right\|^{2}$. If $\lim _{t \rightarrow \infty} E(t)=0$, the complex network achieves cluster synchronization.

For the controlled dynamic network (4.6). Let $\tau=0.1, \xi_{i}=275, i \in J=$ $\{7,8,9,10,11,12,13,28,29,30\}, c=5, \Lambda=0.0934$ in the Theorem 3.1. It can be easily verified that such parameters fits (4.7). 
Figures 2(a), 2(b), and 2(c) give the behavior of $x(t)$, as well as Figure 2(d) which shows how $E(t)$ evolve in pinning united chaotic attractor complex network with initial values chosen randomly in the interval $[-10,10]$.

For the controlled dynamic network (4.6) with adaptive control strength $d_{i}(t)$, let $\Xi^{*}=$ $\Xi$ and keep other parameters the same as selected above, which makes (3.11) correct.

Figure 3 shows the behavior of $x(t)$ and how $E(t)$ evolve in pinning Lorenz chaotic attractor complex network with adaptive control strength and the initial values are chosen randomly in the interval $[-10,10]$.

\subsection{Simulation to Cluster Synchronization with Nonidentical Nodes}

In this simulation, we consider a network with 30 nodes and 3 communities. It is too high and we do not show it out. We give the topology structure Figure 4.

Let $s_{1}(t), s_{2}(t), s_{3}(t)$ be the solution of the uncoupling system $\dot{s}_{i}(t)=f\left(s_{i}(t), t\right)$ with initial values $s_{1}(0)=[1,2,3]^{T}, s_{2}(0)=[4,5,6]^{T}$, and $s_{3}(0)=[7,8,9]^{T}$. Define $E(t)=(1 / 30) \sum_{i=1}^{30}\left\|x_{i}(t)-s_{i}(t)\right\|^{2}$. If $\lim _{t \rightarrow \infty} E(t)=0$, the complex network achieves cluster synchronization.

For the controlled dynamic network (4.6). Let $\tau=0.1, D_{A}=10.2173 I, D_{A}=0.0171 I$, $c=6$, and $\Lambda=0.0071$ in the Theorem 4.1. It can be easily verified that such parameters fits (4.7).

Figures 5(a), 5(b), and 5(c) give the behavior of $x(t)$, as well as Figure 5(d) which shows how $E(t)$ evolve in pinning united chaotic attractor complex network with initial values chosen randomly in the interval $[-10,10]$.

\section{Conclusion}

In the paper, we have investigated the cluster synchronization on pinning control of LCODEs with identical and nonidentical nodes. We give a sufficient condition to make the complex network achieve cluster synchronization. Moreover, adaptive feedback control techniques are used to adjust control strength. Finally, some numerical examples are given, which is essential to verify our theoretical analysis.

\section{Acknowledgments}

The authors are grateful to the editors and the reviewers for their valuable suggestions and comments. This work was supported by Guangdong Education University Industry Cooperation Projects (2009B090300355), Shenzhen Basic Research Project (JC201006010743A), and the 2011 Foundation for Distinguished Young Talents in Higher Education of Guangdong (LYM11115).

\section{References}

[1] S. H. Strogatz and I. Stewart, "Coupled oscillators and biological synchronization," Scientific American, vol. 269, no. 6, pp. 102-109, 1993.

[2] C. M. Gray, "Synchronous oscillations in neuronal systems: mechanisms and functions," Journal of Computational Neuroscience, vol. 1, no. 1-2, pp. 11-38, 1994. 
[3] L. Glass, "Synchronization and rhythmic processes in physiology," Nature, vol. 410, no. 6825, pp. 277$284,2001$.

[4] M. S. De Vieira, "Chaos and synchronized chaos in an earthquake model," Physical Review Letters, vol. 82, no. 1, pp. 201-204, 1999.

[5] L. Kuhnert, K. I. Agladze, and V. I. Krinsky, "Image processing using light-sensitive chemical waves," Nature, vol. 337, no. 6204, pp. 244-247, 1989.

[6] S. Wang, J. Kuang, J. Li, Y. Luo, H. Lu, and G. Hu, "Chaos-based secure communications in a large community," Physical Review E, vol. 66, no. 6, Article ID 065202, 4 pages, 2002.

[7] S. Boccaletti, J. Kurths, G. Osipov, D. L. Valladares, and C. S. Zhou, "The synchronization of chaotic systems," Physics Report, vol. 366, no. 1-2, pp. 1-101, 2002.

[8] Z. Zheng and G. Hu, "Generalized synchronization versus phase synchronization," Physical Review E, vol. 62, no. 6, pp. 7882-7885, 2000.

[9] M. G. Rosenblum, A. S. Pikovsky, and J. Kurths, "From phase to lag synchronization in coupled chaotic oscillators," Physical Review Letters, vol. 78, no. 22, pp. 4193-4196, 1997.

[10] V. N. Belykh, I. V. Belykh, and E. Mosekilde, "Cluster synchronization modes in an ensemble of coupled chaotic oscillators," Physical Review E, vol. 63, no. 3, Article ID 036216, 4 pages, 2001.

[11] M. G. Rosenblum, A. S. Pikovsky, and J. Kurths, "Phase synchronization of chaotic oscillators," Physical Review Letters, vol. 76, no. 11, pp. 1804-1807, 1996.

[12] C. van Vreeswijk, "Partial synchronization in populations of pulse-coupled oscillators," Physical Review E, vol. 54, no. 5, pp. 5522-5537, 1996.

[13] Z. J. Ma, Z. R. Liu, and G. Zhang, "A new method to realize cluster synchronization in connected chaotic networks," Chaos, vol. 16, no. 2, Article ID 023103, 2006.

[14] W. Wu, W. J. Zhou, and T. P. Chen, "Cluster synchronization of linearly coupled complex networks under pinning control," IEEE Transactions on Circuits and Systems I, vol. 56, no. 4, pp. 829-839, 2009.

[15] J. Zhou, X. Wu, W. Yu, M. Small, and J. A. Lu, "Pinning synchronization of delayed neural networks," Chaos, vol. 18, no. 4, Article ID 043111, 2008.

[16] W. L. Lu, B. Liu, and T. P. Chen, "Cluster synchronization in networks of coupled nonidentical dynamical systems.," Chaos, vol. 20, no. 1, Article ID 013120, p. 12, 2010.

[17] W. L. Lu, B. Liu, and T. P. Chen, "Cluster synchronization in networks of distinct groups of maps," European Physical Journal B, vol. 77, no. 2, pp. 257-264, 2010.

[18] K. H. Wang, X. C. Fu, and K. Z. Li, "Cluster synchronization in community networks with nonidentical nodes," Chaos, vol. 19, no. 2, Article ID 023106, 10 pages, 2009.

[19] J. Cao, Z. Wang, and Y. Sun, "Synchronization in an array of linearly stochastically coupled networks with time delays," Physica A, vol. 385, no. 2, pp. 718-728, 2007.

[20] T. P. Chen, X. W. Liu, and W. L. Lu, "Pinning complex networks by a single controller," IEEE Transactions on Circuits and Systems I, vol. 54, no. 6, pp. 1317-1326, 2007.

[21] W. Guo, F. Austin, and S. Chen, "Global synchronization of nonlinearly coupled complex networks with non-delayed and delayed coupling," Communications in Nonlinear Science and Numerical Simulation, vol. 15, no. 6, pp. 1631-1639, 2010.

[22] D. M. Li, J. A. Lu, X. Q. Wu, and G. R. Chen, "Estimating the ultimate bound and positively invariant set for the Lorenz system and a unified chaotic system," Journal of Mathematical Analysis and Applications, vol. 323, no. 2, pp. 844-853, 2006. 


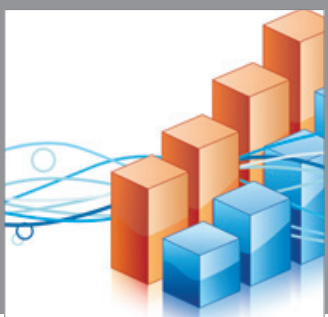

Advances in

Operations Research

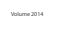

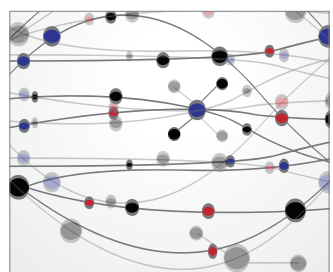

\section{The Scientific} World Journal
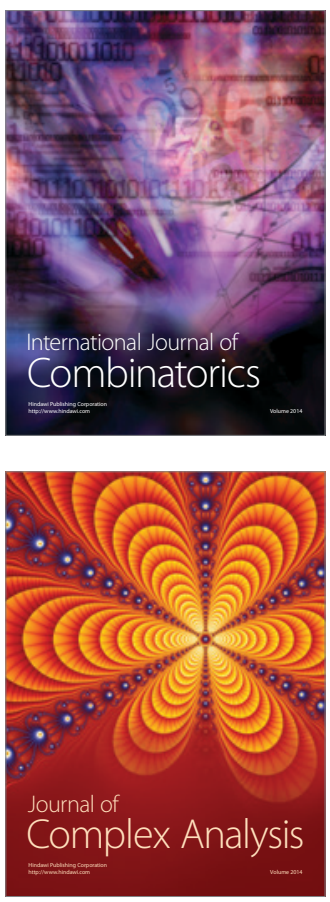

International Journal of

Mathematics and

Mathematical

Sciences
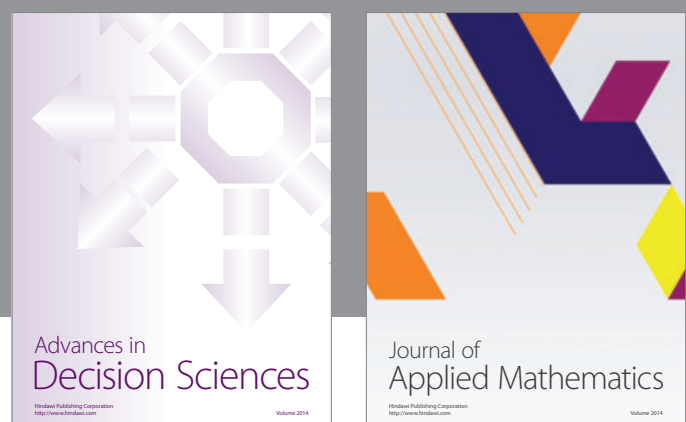

Journal of

Applied Mathematics
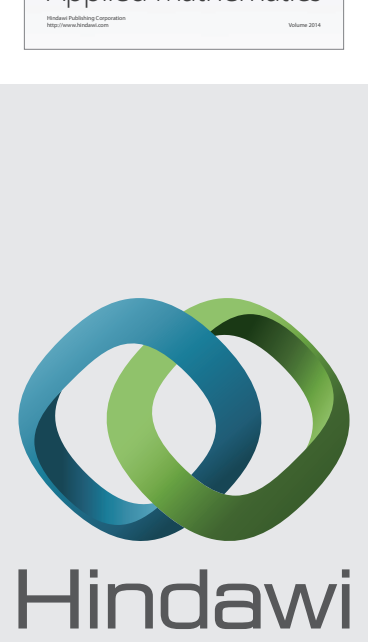

Submit your manuscripts at http://www.hindawi.com
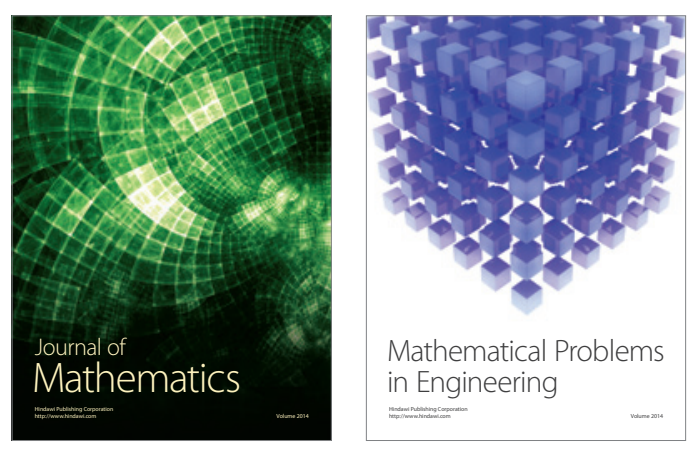

Mathematical Problems in Engineering
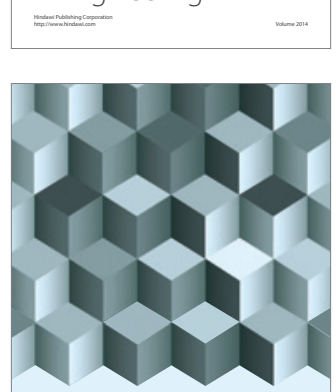

Journal of

Function Spaces
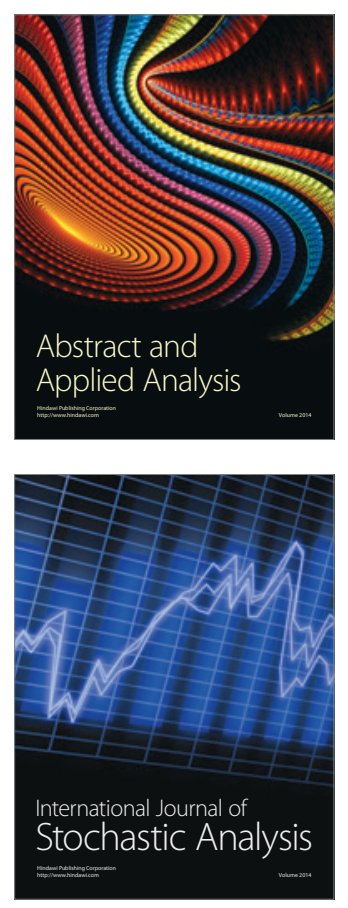

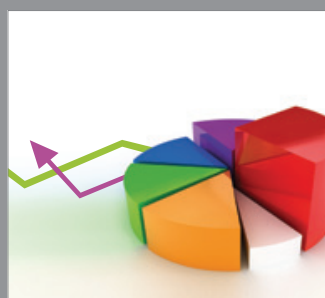

ournal of

Probability and Statistics

Promensencen
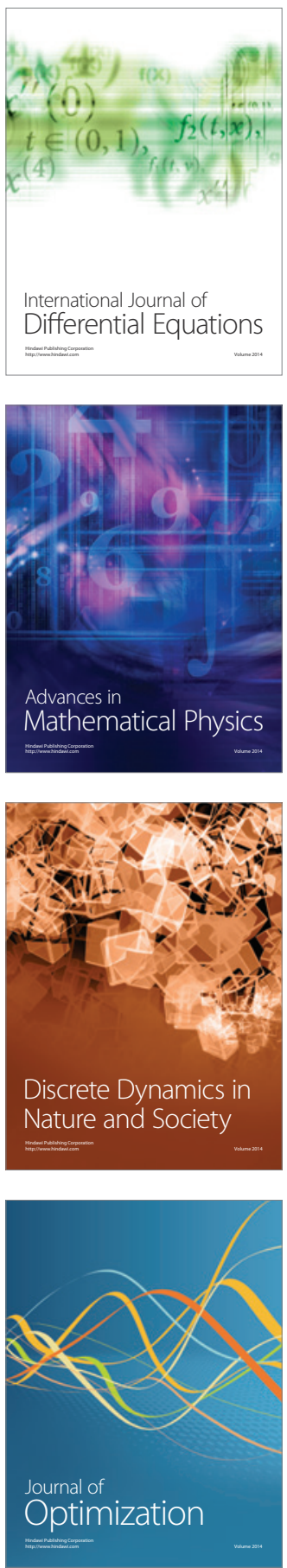